\title{
Vasculitis asociada al consumo de cocaína adulterada con levamisol
}

Vasculitis related to levamisole adulterated cocaine consumption

\author{
Guillermo González Rodríguez ${ }^{1}$, Carlos Guillermo Sánchez², Jasson Sanclemente \\ 1. Médico dermatólogo, Fundación Hospital San José, Guadalajara de Buga; Facultad de Medicina, Universidad Libre, Cali, Colombia \\ 2. Director médico, Fundación Hospital San José, Guadalajara de Buga, Colombia \\ 3. Médico interno, Facultad de Medicina, Universidad Libre, Cali; Fundación Hospital San José, Guadalajara de Buga, Colombia
}

\section{RESUMEN}

Se presenta el caso de un paciente de 41 años de edad, natural de Cali y procedente de Guadalajara de Buga, que presentaba gran cantidad de lesiones ulceradas recubiertas por costras hemorrágicas seropurulentas y fétidas, que comprometían los miembros inferiores, y lesiones necróticas, purpúricas y de diferentes diámetros, en la piel del resto del cuerpo. Era fumador crónico y consumidor habitual de cocaína, motivo por el cual se hospitalizó con el diagnóstico de úlceras crónicas sobreinfectadas y posible vasculitis por consumo de cocaína.

PALABRAS CLAVE: vasculitis, cocaína, coca, levamisol.

\section{SUMMARY}

The case of a 41 year-old patient from Cali, living in Guadalajara de Buga, is presented. The individual presented with several ulcers covered by fetid sero-purulent haemorrhagic scars in the inferior limbs, and necrotic purpuric lesions of different sizes in the rest of the skin. The patient is also a chronic smoker and regular cocaine user; he was hospitalized with the diagnoses of over-infected chronic ulcers and possible vasculitis due to cocaine consumption.

KEY WORDS: Vasculitis, cocaine, coca, levamisole.

\section{INTRODUCCIÓN}

El consumo de sustancias psicoactivas en Colombia, cualquiera que ella sea (marihuana, cocaína, bazuco, éxtasis o heroína), se ha aumentado como lo demuestra el Estudio Nacional de Consumo de Sustancias Psicoactivas en Colombia, donde se comparan las cifras estadísticas del 2008 con las del 2013; la cocaína ocupa el segundo lugar entre las sustancias ilícitas de mayor consumo en Colombia ${ }^{(1)}$. El consumo de cocaína se ha asociado con numerosas manifestaciones sistémicas, entre ellas, lesiones cutáneas vasculíticas.

Se describen los hallazgos clínico-patológicos que se encontraron en un paciente adulto, consumidor habitual de cocaína, atendido en el Servicio de Urgencias y,
Correspondencia:

Guillermo González Rodríguez

Email:

guillermo0250@live.com

Recibido: 10/07/2016

Aceptado: 02/11/2016

Conflictos de interés:

No se reportan conflictos de interés.

Financiación:

Ninguna. 
posteriormente, hospitalizado en la Fundación Hospital San José de Guadalara de Buga en el 2016.

\section{CASO CLÍNICO}

Se trata de un paciente de sexo masculino de 41 años de edad, con antecedentes de toxicomanía por consumo de cocaína y tabaquismo desde los 21 años, que consultó por un cuadro clínico de dolor intenso en los miembros inferiores, el abdomen y la región lumbar.

En el momento del ingreso, el paciente presentaba bajo peso, facies de dolor, signos vitales normales y las mucosas con signos de deshidratación.

En el examen físico se encontraron úlceras grandes y fétidas, que comprometían el tercio medio, el distal y los lados de ambas piernas, y lesiones necróticas secas y muy dolorosas en el tronco (figuras 1 a 3). Con la sospecha clínica de úlceras crónicas por vasculitis secundaria al consumo de cocaína, se decidió hospitalizarlo.

En los estudios complementarios de laboratorio, se encontró: proteína C reactiva elevada $(58,97 \mathrm{mg} / \mathrm{L})$; Pseudomonas aeruginosa y Klebsiella pneumoniae, en el cultivo de la secreción de ambas pantorrillas; título de anticuerpos antinucleares (ANA) de 1:320; título de anticuerpos citoplásmicos antineutrófilos (c-ANCA/p-

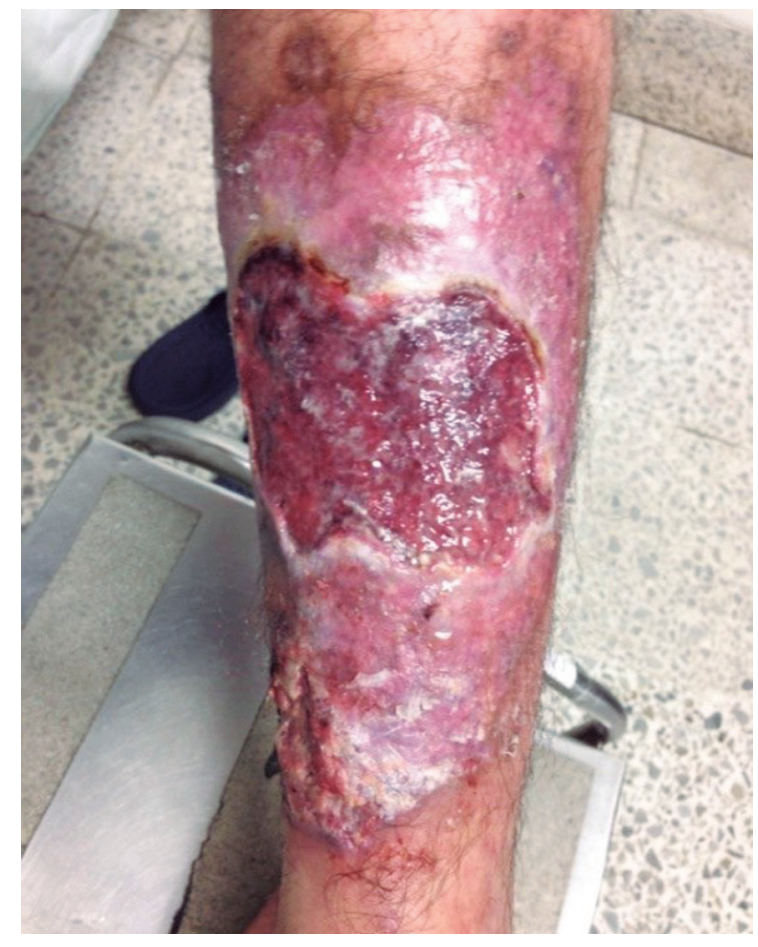

Figura 1. Al ingreso a urgencias, se observaron úlceras grandes y fétidas, que comprometían el tercio medio de la pierna derecha.
ANCA) (cytoplasmic-ANCA/perinuclear-ANCA), de 1:160; velocidad de sedimentación globular (VSG) de 62 $\mathrm{mm}$ por hora; trombocitosis de 855.000 plaquetas por $\mu \mathrm{l}$; y aminotransferasa de aspartato (TGO), $65 \mathrm{UI} / \mathrm{L}$.

Las pruebas para HIV, la de reagina plasmática rápida (RPR), la de crioglobulinas, el hemocultivo, el antígeno de superficie de la hepatitis $\mathrm{B}$ y el de la hepatitis $\mathrm{C}$ fueron negativos; los fosfolípidos, los anticuerpos IgG e IgM, las fracciones $\mathrm{C}_{3}$ y $\mathrm{C}_{4}$ del complemento y la fosfatasa alcalina estuvieron dentro de límites normales. En la radiografía de tórax, se observaron cambios por bronquitis crónica y, en la biopsia de piel, infiltrados perivasculares de polimorfonucleares y trombosis (figuras 4 y 5). El examen toxicológico de orina no se practicó oportunamente por trabas administrativas.

Se hizo el diagnóstico diferencial con las vasculitis con anticuerpos citoplásmicos antineutrófilos (ANCA) positivos (granulomatosis de Wegener, síndrome de ChurgStrauss, enfermedad de Kawasaki, poliangeítis microscópica y púrpura de Henoch-Schönlein), las cuales se descartaron por la historia clínica -dado que el paciente refirió ser consumidor habitual de cocaína-, por los análisis complementarios de laboratorio y por la biopsia de piel $^{(2)}$.

El abuso de cocaína produce una gran cantidad de ma-

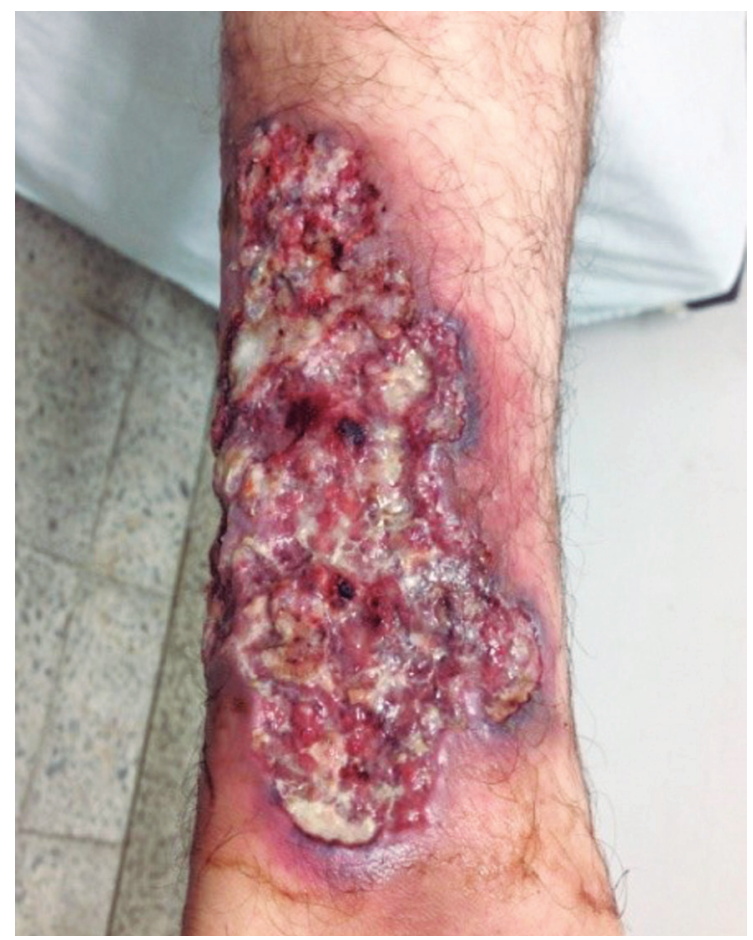

Figura 2. Al ingreso a urgencias, se observaron úlceras grandes y fétidas, que comprometían el tercio distal de la pierna izquierda. 


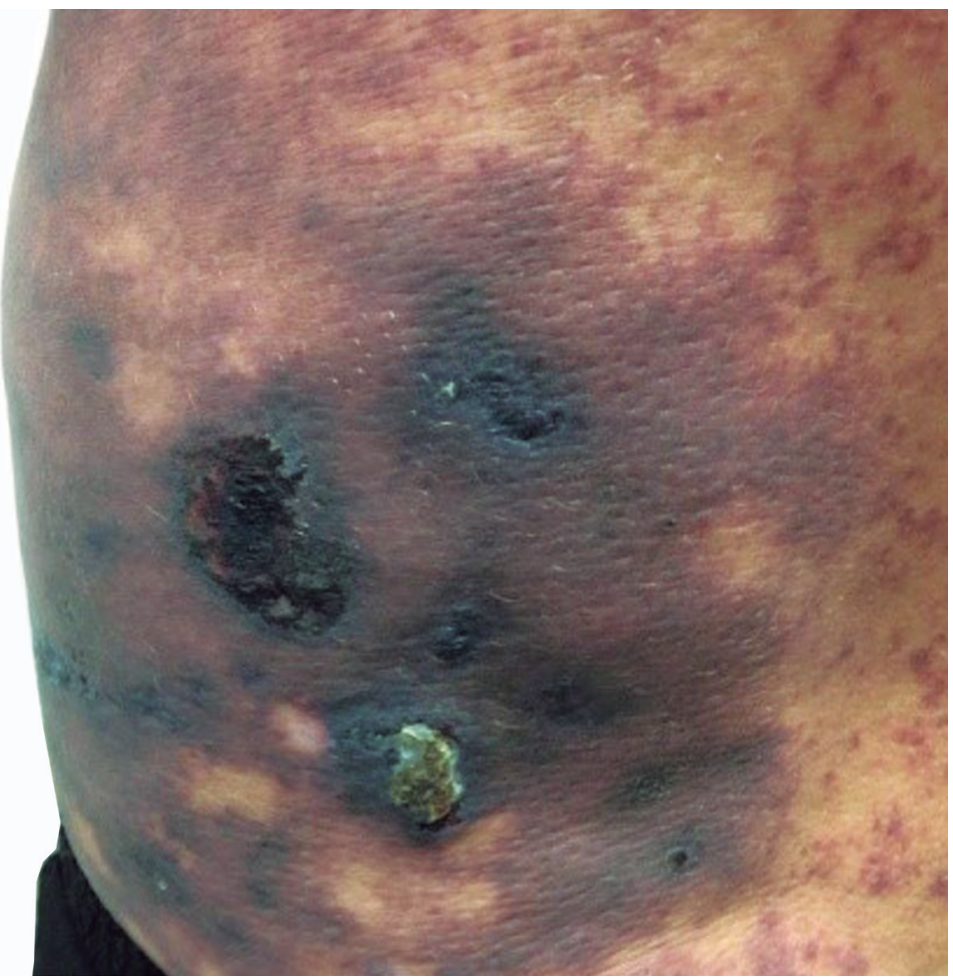

Figura 3. Al ingreso a urgencias, se observaron lesiones necróticas secas y muy dolorosas en el tronco.

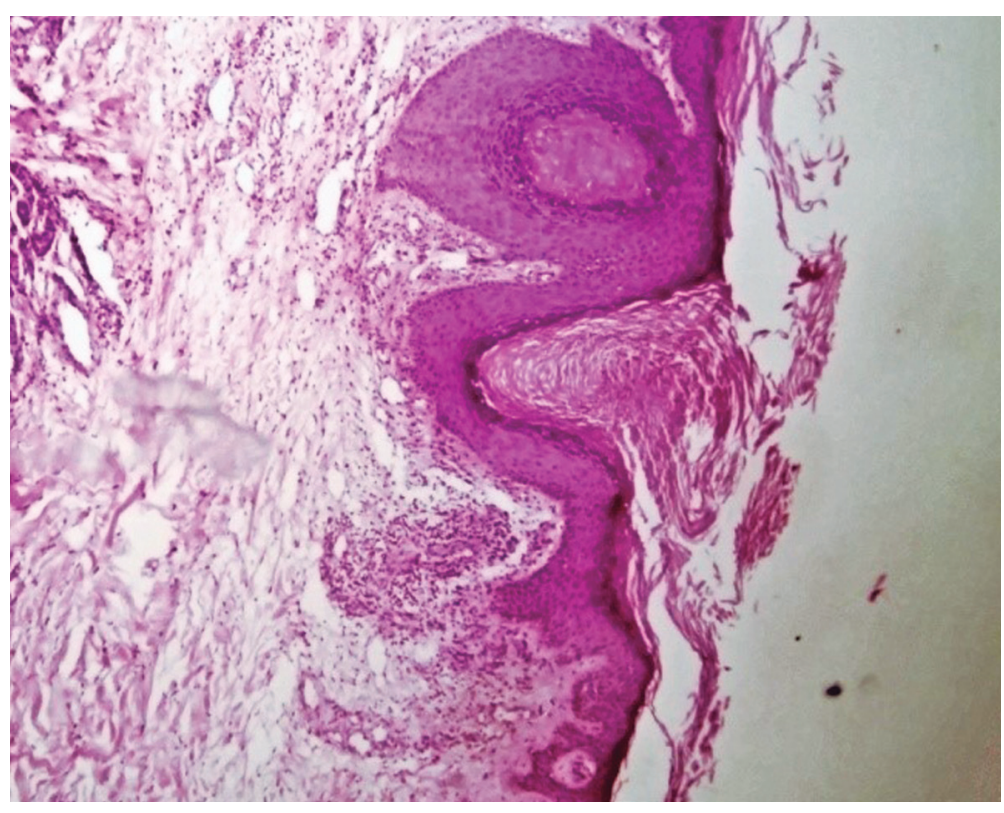

Figura 4. Tapón de queratina, hiperqueratosis ortoqueratósica con acantosis epitelial e infiltrado perivascular mixto. Hematoxilina y eosina, 4X.

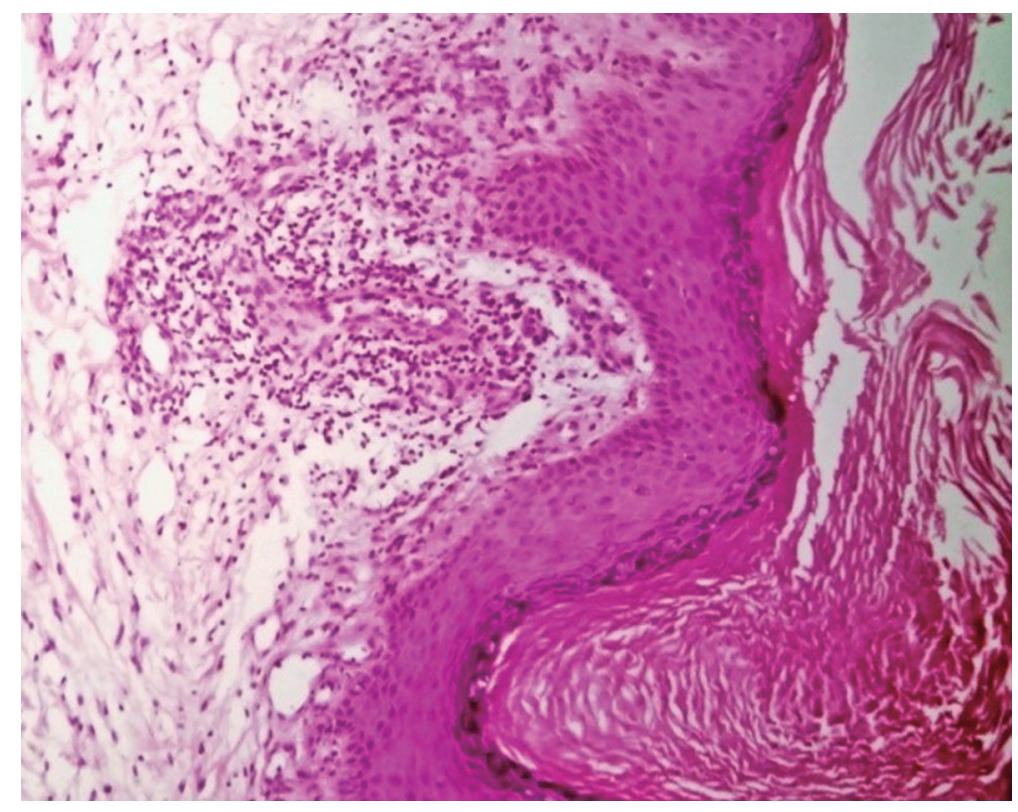

Figura 5. A mayor aumento, infiltrado inflamatorio constituido principalmente por polimorfonucleares neutrófilos en vasos superficiales y profundos. Hematoxilina y eosina, $10 \mathrm{X}$. 
nifestaciones cutáneas que pueden ir desde formas muy graves con úlceras crónicas en la piel e infartos digitales, hasta el síndrome de Stevens-Johnson. Puede inducir lesiones destructivas de la línea media que pueden simular una vasculitis con ANCA positivos, como la granulomatosis con poliangeítis, o conllevar una perforación del tabique nasal, como muy bien ha sido descrito. La púrpura retiforme, en su presentación típica, compromete bilateralmente las mejillas, las orejas, la nariz y el paladar duro de los drogadictos consumidores de cocaína adulterada con levamisol; su cuadro histopatológico es el de una microtrombosis vascular y vasculitis leucocitoclástica de pequeños vasos, el cual estuvo ausente en el presente caso ${ }^{(3)}$.

La desmorfina, o krokodil, es una de las sustancias alucinógenas más frecuentemente consumida en el norte de Europa y en los países de la antigua Unión Soviética, pero con una rápida expansión a los Estados Unidos y Suramérica; conlleva una fuerte adicción, es de bajo costo y produce efectos inmediatos. Por motivos económicos, el consumo de opio y heroína en estos países europeos ha disminuido notablemente pero, al mismo tiempo, se reporta un incremento del consumo de sustancias que contienen codeína. La molécula del krokodil, muy similar a la de la morfina, es un potente $\mu$-opioide con gran toxicidad y un poder analgésico 5 a 10 veces mayor que el de la morfina; se sintetiza a partir de la codeína combinada con otros aditivos de fácil obtención y bajo costo.

El neologismo krokodil (del ruso cocodrilo) se originó a partir de la apariencia de piel de cocodrilo que pueden presentar los consumidores, con piel verdosa oscura y con descamación ${ }^{(4,5)}$.

Con el diagnóstico clínico-patológico de vasculitis por consumo de cocaína adulterada, posiblemente con levamisol, se inició el tratamiento. Hoy en día, no hay consenso sobre el tratamiento de los pacientes con estas manifestaciones. En este caso, se suspendió el consumo de cocaína y se hizo un manejo multidisciplinario con los servicios de psiquiatría, cirugía plástica, medicina interna y dermatología.

El paciente recibió tratamiento con alprostadil (prostaglandina E1) como vasodilatador periférico, $80 \mu \mathrm{g}$ intravenosos diarios durante cuatro semanas; clindamicina, una ampolla de $600 \mathrm{mg}$ cada seis horas durante siete días, y ceftriaxona, $1 \mathrm{~g}$ cada 12 horas durante siete días; prednisona, $75 \mathrm{mg}$ una toma al día durante seis días; piperacilina-tazobactam, una ampolla de 4,5 $\mathrm{g}$ por vía intravenosa cada seis horas, durante siete dias; y levomepromazina, $40 \mathrm{mg} / \mathrm{ml}$, 10 gotas cada 24 horas por vía oral. Se le administró suplemento nutricional por vía oral. Se manejó con curaciones por parte de terapia enterostomal, tratamiento médico y de enfermería, durante su hospitalización, con resultados clínicos favorables.

Dos meses y medio después de su egreso, el paciente asistió a control con dermatología; se observó clara mejoría clínica, con procesos de cicatrización en las pantorrillas, el abdomen y la región lumbar (figuras 6 y 7).

\section{REVISIÓN DE TEMA}

La cocaína, conocida como la benzoilmetilecgonina, es una sustancia alcaloide obtenida de las hojas del arbusto Erytroxylon coca. La planta es originaria de los países de la región andina de América ${ }^{(6)}$.

La hoja de coca posee 14 alcaloides naturales que son: cocaína, egnonina, atropina, pectina, papaína, higrina, globulina, piridina, quinolina, conina, cocamina, reserpina, benzoína e inulina ${ }^{(7)}$. Estos 14 alcaloides, los aminoácidos, los ácidos y las vitaminas A, B1, B2, C y $\mathrm{E}$, la tiamina, la niacina, la riboflavina y otros tantos productos, convierten a la planta de coca en una de las más completas del mundo en nitrógeno no proteico, que elimina las toxinas y agentes patógenos del cuerpo hu$\operatorname{mano}^{(7)}$.

La cocaína inhibe la recaptación de serotonina en las terminales sinápticas, como consecuencia del aumento de la serotonina y del paso de sustancias simpaticomiméticas a través de la barrera hematoencefalica lesionadas por cifras de tensión arterial elevadas de manera súbita. La cocaína puede producir vasoconstricción intensa, sobre todo en las arterias de mediano y gran calibre. Los fenómenos hemorrágicos se relacionan con crisis hipertensivas, ruptura de malformaciones aneurismáticas o transformación hemorrágica de lesiones isquémicas ${ }^{(8)}$. En la actualidad, la cocaína se considera un factor de riesgo independiente, tanto para eventos isquémicos como hemorrágicos, sin importar la frecuencia o la duración de su consumo ${ }^{(8)}$.

Los mecanismos responsables del daño vascular asociado al uso de cocaína son múltiples y no están totalmente dilucidados. Principalmente, debido a sus propiedades simpaticomiméticos, aumenta el consumo de oxígeno, produce taquicardia, eleva la presión arterial, aumenta la contractibilidad miocárdica e induce vasoconstricción coronaria. Sin embargo, los efectos vasomotores no explican todas las complicaciones isquémicas que se observan en estos pacientes, especialmente los que se presentan a largo plazo ${ }^{(9)}$.

Las evidencias obtenidas de los estudios in vitro y en modelos animales demuestran que la cocaína induce disfunción de las células endoteliales que inician el 


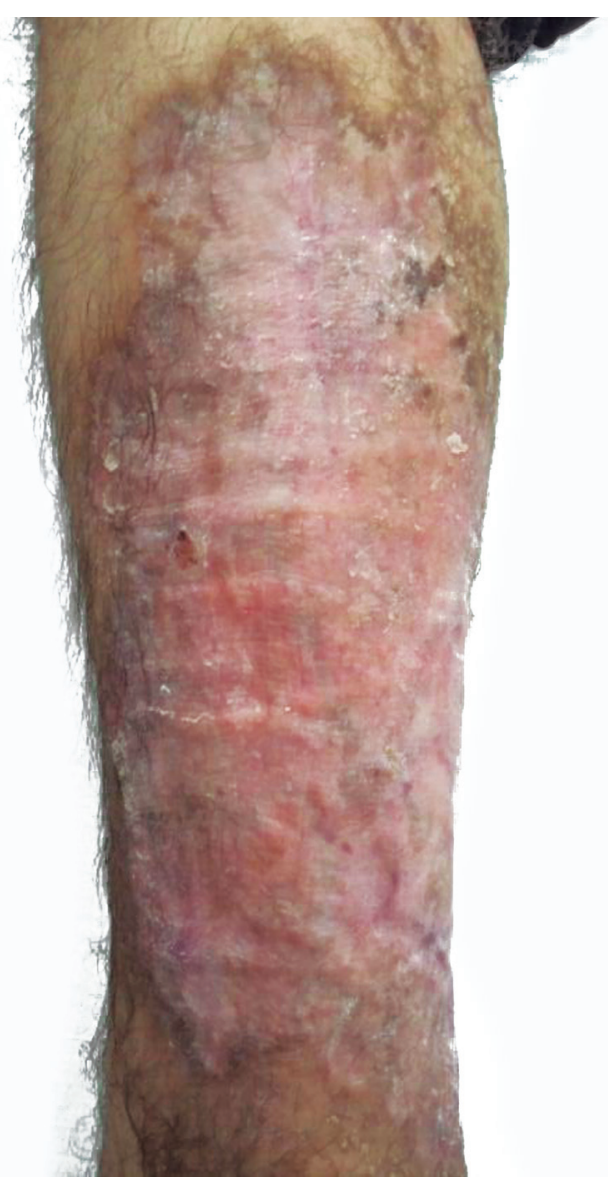

Figura 6. Pantorrilla derecha, 75 días después del egreso hospitalario

daño vascular aterosclerótico y esta disfunción endotelial se mantiene por más de un mes de abstinencia estricta, lo que sugiere que el daño vascular inducido por la droga es a largo plazo y no solamente asociado a los efectos agudos de la cocaína. En este mismo sentido, y en relación con las complicaciones trombóticas de la adicción, se ha demostrado activación de las plaquetas tanto en relación con el uso agudo como crónico de la cocaína ${ }^{(9,10)}$.

La cocaína se ha asociado con numerosas manifestaciones clínicas en la piel, que incluyen pseudovasculitis, urticaria vasculítica, necrosis cutánea multifocal aguda, vasculitis de Churg-Strauss, úlceras crónicas, fenómeno de Raynaud, vasculitis necrosante granulomatosa, perforación nasal del tabique, púrpura de Henoch-Schönlein, granuloma de la línea media, pustulosis exantemática generalizada aguda, vasculitis necrosante, síndrome de Stevens-Johnson, enfermedades ampollosas y enfermedad de Buerger. Sin embargo, no se pueden excluir totalmente otras causas de manifes-

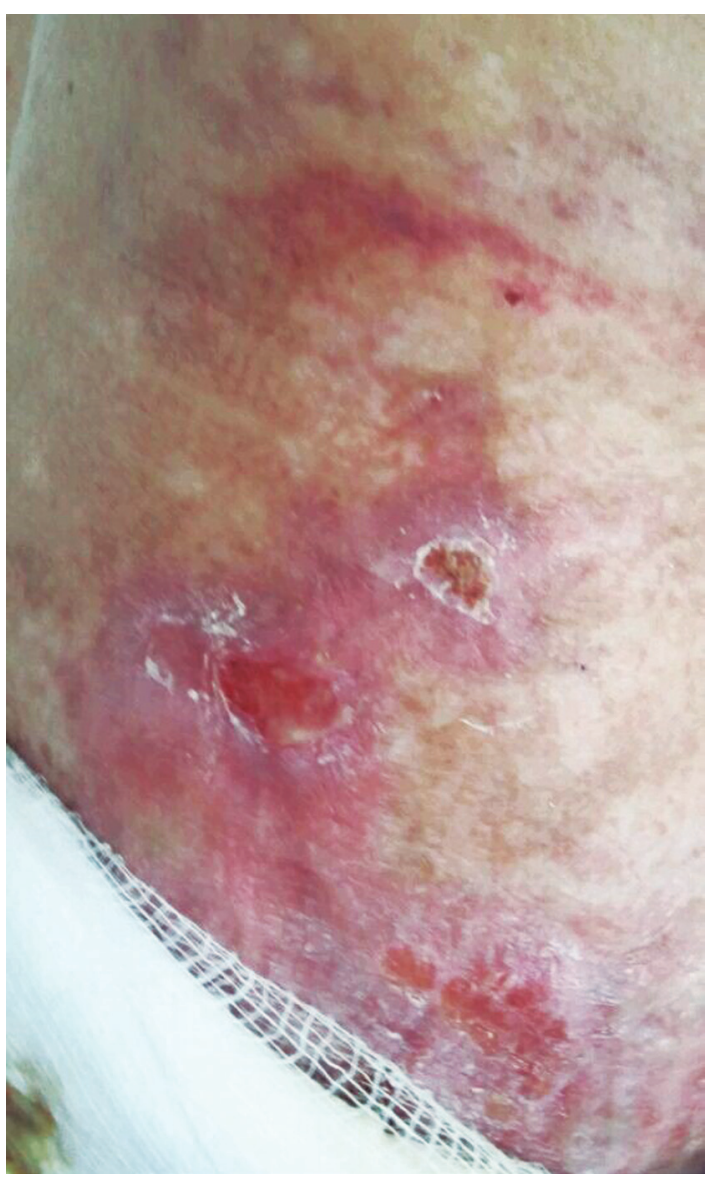

Figura 7. Aspecto del tronco, 75 días después del egreso hospitalario

taciones en la piel aun cuando se encuentren otras sustancias ilícitas en el examen toxicológico. Un resultado positivo no siempre indica que sea la causa de una manifestación cutánea ${ }^{(11-13)}$.

Debido a que el consumo de drogas ilícitas va en ascenso y a que estas causan muchas manifestaciones cutáneas, debería considerarse la posibilidad diagnóstica de erupción inducida por drogas ilícitas a aquellas lesiones en la piel que se asocien con un comportamiento extraño, historia clínica vaga, hallazgos negativos en valoraciones previas acompañados de signos de delirio, parasitofobia $u$ otras condiciones psiquiátricas ${ }^{(14)}$.

Además del problema de consumo, en la actualidad, se ha visto que se añaden distintos sustratos con los que se logra adulterar la droga; esas sustancias son: bicarbonato, benzocaína, harina, hidroxicina, diltiazen, lactosa, soda, sal, levamisol, etc. Estas sustancias, conocidas como "agentes para cortar la droga", son adicionadas en cualquier etapa de la producción, empaque o distribución ${ }^{(7)}$. 
La cocaína adulterada con levamisol se ha detectado desde 2003 y la incidencia de toxicidad con cocaínalevamisol se ha incrementado rápidamente desde el $2008^{(15)}$. La adulteración con levamisol le da a la cocaína un efecto similar, complementario o potencializador de la droga con un compuesto más barato, además de elevar los efectos euforizantes; los efectos secundarios son mínimos en comparación con otras sustancias ${ }^{(7,15)}$. El levamisol es un medicamento antiparasitario de amplio espectro, de uso veterinario para el tratamiento de nematodos gastrointestinales y pulmonares, principalmente en bóvidos, óvidos y cerdos. Se ha utilizado en humanos durante muchos años como terapia adjunta para procesos infecciosos crónicos, autoinmunitarios, estados de inmunosupresión, incluso en aquellos asociados con neoplasias malignas. Son varios los reportes sobre su utilización en el tratamiento de verrugas vulgares con buenos resultados y, desde 1977, se conoció su importancia en la inmunoterapia del cáncer, principalmente en el cáncer de colon ${ }^{(16)}$. En las artritis crónicas de tipo reumatoideo, el levamisol se ha utilizado por sus efectos inmuno-estimulantes duraderos ${ }^{(17)}$. En el síndrome nefrótico, la utilización de levamisol se ha incorporado al esquema de tratamiento de estos pacientes ${ }^{(18)}$. $\mathrm{Su}$ absorción es rápida, alcanzando concentraciones plasmáticas máximas en 1 a 2 horas, con una vida media aproximada de 5 a 6 horas y, además, posee efecto acumulativo. Su uso en humanos se descontinuó a partir del 2005 ya que se comprobó que cuando se administraba por periodos prolongados podía dar lugar a la aparición de efectos secundarios, principalmente hematológicos (agranulocitosis, trombopenia y neutropenia). Estos efectos dependen de la dosis y son reversibles; su aparición se asocia con la presencia del antígeno mayor de histocompatibilidad HLA-B27, lo que sugiere que estos pacientes pueden estar genéticamente predispuestos a desarrollar estas complicaciones hematológicas, y con la presencia de ANCAS positivos ${ }^{(7,16,19)}$.

El síndrome cocaína-levamisol se caracteriza por manifestaciones cutáneas, vasculopatías, agranulocitosis y leucoencefalopatía inflamatoria reversible. Característicamente, el cuadro clínico mejora con la suspensión del consumo de la sustancia.

En el 2012, Larocque y Hoffman recopilaron 203 casos de pacientes consumidores de cocaína adulterada con levamisol, y describieron que las dos complicaciones principalmente encontradas eran la neutropenia (140 casos) y las lesiones dermatológicas (84 casos). Además, se le relaciona con un cuadro de malestar general y artralgias de las grandes articulaciones hasta en el 16,7 \% de los $\operatorname{casos}^{(20)}$.

El compromiso cutáneo es tan variado que incluye erupciones liquenoides y vasculitis cutáneas caracterizadas por la aparición de lesiones purpúricas en placas y ampollas hemorrágicas, dolorosas, que aparecen de repente en cualquier zona del cuerpo, pero con especial predilección por las extremidades inferiores, la nariz y las orejas. Las lesiones pueden progresar a necrosis secas o úlceras, y complicarse con sobreinfecciones ${ }^{(19,20)}$. Se han descrito episodios febriles con evidencias patológicas de liberación de histamina y granulación de mastocitos que sugieren que el levamisol puede desencadenar reacciones de hipersensibilidad de tipo I-III, como también presentarse una falla renal de tipo pauciinmunitario focal necrosante, hemorragia pulmonar e hiponatremia, sin entenderse muy bien el mecanismo fisiopatológico implicado ${ }^{(19)}$.

La singularidad de las vasculitis en el espectro del consumo de cocaína insinúa al levamisol como un posible culpable, teniendo en cuenta que, actualmente, más del $70 \%$ de la oferta de cocaína en Estados Unidos viene adulterada con este medicamento ${ }^{(14)}$.

Se han considerado varios mecanismos de acción de este complejo cocaína-levamisol para producir estos efectos vasculíticos tan agresivos y diversos sobre la piel y otros órganos, entre ellos ${ }^{(21)}$ : el depósito de complejos inmunitarios en los neutrófilos, con la consiguiente activación de la vía del complemento y la lisis celular final, efectos inmunomoduladores en la producción de autoanticuerpos antineutrófilos; presencia de complejos inmunitarios que median las vasculitis con depósitos vasculares de IgM, IgA, IgG y C3 y tinción para fibrina, y supresión de la médula espinal.

Desde el punto de vista inmunológico, tanto la vasculopatía cutánea como la leucopenia se han asociado a la aparición de anticuerpos anticitoplásmicos de neutrófilos (ANCA) al igual que anticuerpos antifosfolípidos y ANA negativos. La presencia de estos anticuerpos se da por la exposición en el tiempo a estas sustancias, por lo que no se espera su presencia en el consumo agudo y se ha descrito la normalización de los anticuerpos en 2 a 14 meses después de la cesación del consumo ${ }^{(15)}$.

El protocolo de estudio de una vasculitis inducida por cocaína es idéntico al de las vasculitis inducidas por fármacos; incluye hemograma, velocidad de sedimentación globular, bioquímica general, VDRL, HIV, radiografía de tórax, análisis de orina, pruebas de función hepática y sangre oculta en heces. Posteriormente, se deben considerar otros estudios más específicos, como las biopsias de piel con inmunoflorescencia directa o sin ella, anticuerpos anticardiolipina, niveles de proteína C, $\mathrm{S}$ y crioglobulinas. Se deben determinar niveles de ANCA, ANA, factor reumatoide, $\mathrm{C}_{3}-\mathrm{C}_{4}, \mathrm{y}$ anticuerpos para la hepatitis B y la $\mathrm{C}^{(12)}$. 
La benzoilecgonina puede detectarse en plasma hasta 24 horas después de su administración ${ }^{(6)}$.

\section{CONCLUSIÓN}

El consumo de drogas ilícitas en Colombia está en aumento ${ }^{(1)}$. La adulteración de la cocaína con levamisol es una realidad que está causando enfermedad en la población consumidora, siendo frecuente la vasculopatía cutánea, por lo que se debe hacer énfasis en preguntar sobre el consumo de sustancias psicoactivas para poder hacer adecuados diagnósticos diferenciales.

\section{AGRADECIMIENTOS}

A Iván E. Bravo Naranjo, por su ayuda con el estudio histopatológico de este paciente.

\section{REFERENCIAS}

1. Comisión Asesora para la Política de Drogas en Colombia. Lineamientos para un nuevo enfoque de la política de drogas en Colombia. Bogotá: Comisión Asesora para la Política de Drogas en Colombia; 2015. p. 78.

2. Vásquez L. Vasculitis cutáneas. Rev Asoc Colomb Dermatol. 2000;8:149-60.

3. Formeister EJ, Falcone MT, Mair EA. Facial cutaneous necrosis associated with suspected levamisole toxicity from tainted cocaine abuse. Ann Otol Rhinol Laryngol. 2015;124:30-4.

4. Baquero-Escribano A, Beltrán-Negre MT, CalvoOrenga G, Carratalá- Monfort S, Arnau-Peiró F, Meca-Zapatero S, et al. Consumo de krokodil por vía oral en España: a propósito de un caso. Adicciones. 2016;28:242-5.

5. Haskin A, Kim N, Aguh C. A new drug with a nasty bite: A case of krokodil-induced skin necrosis in an intravenous drug user. JAAD Case Reports. 2016;2:174-6.

6. Mosquera JT, Menéndez MC. Efectos toxicológicos y neuropsiquiátricos producidos por consumo de cocaína. Rev Fac Med Univ Nac Colomb. 2005;53:10-26.

7. Núñez V. Vasculitis cutánea leucocitoclástica y glomeruloesclerosis focal y segmentaria con formación de semilunas en una joven consumidora de cocaína: crack cortada con levamisol V. Med Leg Costa Rica. 2012;29:103-12.

8. Larrosa-Campo D, Ramón-Carbajo C, Benavente-
Fernández L, Álvarez-Escudero R, Zeidan-Ramón N, Calleja-Puerta S, et al. Diagnóstico del ictus por cocaína y sus complicaciones. Rev Neurol. 2013;57:167-70.

9. Toro MF. La drogadicción y el sistema inmunológico. IATREIA. 1989;2:122-7.

10. Massardo T, Pino Á, Berrocal I, Castro G, Prat H, Pereira J. Daño vascular asociado a uso de cocaína: Caso clínico. Rev Med Chil. 2012;140:50711.

11. Brewer JD, Meves A, Bostwick JM, Hamacher KL, Pittelkow MR. Cocaine abuse: Dermatologic manifestations and therapeutic approaches. J Am Acad Dermatol. 2008;59:483-7.

12. Salas-Espíndola Y, Peniche-Castellanos A, López-Gehrke I, Mercadillo-Pérez P. Vasculitis leucocitoclástica asociada a consumo de cocaína. Actas Dermosifiliogr. Actas Dermo-Sifiliográficas. 2011;102:825-7.

13. Neynaber S, Mistry-Burchardi N, Rust C, Samtleben W, Burgdorf WHC, Seitz MA, et al. PR3ANCA-positive necrotizing multi-organ vasculitis following cocaine abuse. Acta Derm Venereol. 2008;88:594-6.

14. López V. Vasculitis asociada al consumo de cocaína adulterada. Rev Fármacos. 2012;21:5-10.

15. Chung C, Tumeh PC, Birnbaum R, Tan BH, Sharp L, McCoy E, et al. Characteristic purpura of the ears, vasculitis, and neutropenia-a potential public health epidemic associated with levamisole-adulterated cocaine. J Am Acad Dermatol. 2011;65:722-5.

16. González G, Trujillo R. Condilomas acuminados en niños. Rev Asoc Colomb Dermatol. 1999;7:33-7.

17. Sierra AF. Tratamiento farmacológico de las enfermedades reumáticas. El Peu. 2006;26:32-42.

18. Comité de Nefrología, Sociedad Argentina de Pediatría. Consenso de tratamiento del síndrome nefrótico en la infancia. Arch Argent Pediatr. 2014;112:277-84.

19. Lee KC, Ladizinski B, Federman DG. Complications associated with use of levamisolecontaminated cocaine: An emerging public health challenge. Mayo Clin Proc. 2012;87:581-6.

20. Garcés J, Berrouet MC, Velásquez MC. Presentación de tres casos de síndrome cocaínalevamisol en Medellín. Rev CES Med. 2015;29:295304.

21. Ramos-Aliaga R. Fraccionamiento químico de la hoja de coca y obtención de un producto rico en proteínas. Revista de la Sociedad Química del Perú. 2005;71:3-11. 